\title{
The strongest magnetic fields in the universe: how strong can they become?
}

\author{
Rudolf A. Treumann ${ }^{1,2 *}$, Wolfgang Baumjohann ${ }^{3}$ and André Balogh ${ }^{4}$ \\ ${ }^{1}$ International Space Science Institute Bern, Bern, Switzerland \\ ${ }^{2}$ Geophysics Department, Munich University, Munich, Germany \\ ${ }^{3}$ Extraterrestrial Physics, Space Research Institute, Austrian Academy of Sciences, Graz, Austria \\ ${ }^{4}$ The Blackett Laboratory, Imperial College London, London, UK
}

\section{Edited by:}

Vladislav Izmodenov, Space

Research Institute, Russia

Reviewed by:

Mikhail V. Medvedev, University of Kansas, USA

Sergey Moiseenko, Space Research institute, Russia

*Correspondence:

Rudolf A. Treumann, ISSI Bern, Hallerstrasse 6, CH-3012 Bern,

Switzerland

e-mail: rudolf.treumann@geophysik. uni-muenchen.de
Magnetic fields in the universe are in general weak, of the order of $\mu$ Gauss only. However, in compact objects they assume extraordinarily large values. These are produced by gravitational collapse of massive magnetized objects. Clearly, fields in the massive progenitor are energetically limited by the available energy which can be fed into the generation of currents and magnetic fields. However, when collapsing down to small scales magnetic fields become superstrong exceeding any limits which can be reached in the laboratory. A brief review and discussion is given on the absolute limitation to the magnetic field strengths which can be obtained during such collapses.

Keywords: strong magnetic fields, neutron stars, pulsars, magnetars, flux quanta, quantum limit, Aharonov-Bohm-scaling, electron radius

\section{INTRODUCTION}

The large-scale dynamics of the universe is governed by the general cosmic expansion and the gravitational field of the massive objects. Magnetic fields are believed not to play any major role in the former $[1,2]$. It is believed that magnetic fields have not, or at least not at appreciable strength, been present at the Big Bang and during the following inflationary period. If they were present at all then in the form of the spurious magnetic monopoles. They become important on smaller scales. On scales of compact magnetized objects they start becoming non-negligible and, for a number of processes [3], even become the dominant force.

Magnetic fields are bound to electric current flow and thus, in contrast to electric fields whose sources are elementary charges and charge differences, be generated by processes which cause electric currents. Currents imply non-ambipolar transport of charges. The question of how strong magnetic fields can become is thus reduced to the question of how strong any currents can become. In classical electrodynamics this implies from Ampère's law for stationary magnetic fields that

$$
\nabla \times \mathbf{B}=\mu_{0} \mathbf{J}, \quad \mathbf{J}=e\left(N_{i} \mathbf{V}_{i}-N_{e} \mathbf{V}_{e}\right) \approx-e N\left(\mathbf{V}_{e}-\mathbf{V}_{i}\right)
$$

if restricting to charge transport alone and assuming nonmagnetic media of (for simplicity singly charged) ion and electron densities and bulk velocities $N_{i, e}, \mathbf{V}_{i, e}$ respectively. Otherwise one would add a magnetization term $\mathbf{M}$ which depends on the properties of matter. Determination of $\mathbf{M}$ requires a quantum mechanical treatment in the framework of solid state physics.

Assuming, without restriction, quasineutrality $N_{e} \approx N_{i}=N$, only velocity differences contribute. Since electrons are substantially more mobile than ions, the current can reasonably be approximated by the electron current $\mathbf{J} \approx-e N \mathbf{V}_{e}$, a condition strictly holding in the ion frame of reference. Since velocities are limited by the velocity of light $c$, the magnetic field is classically limited by

$$
\nabla \times \mathbf{B}<\mu_{0} e N c, \quad \text { or } \quad B<\mu_{0} e N c L \approx 6 \times 10^{-8} N_{\mathrm{cc}} L_{\mathrm{km}}
$$

suggesting that the magnetic field grows with $L$ and density $N$. Here $N_{\mathrm{cc}}$ is in units of electrons per $\mathrm{cm}^{-3}$, and $L_{\mathrm{km}}$ is the length scale across a current filament in units of $\mathrm{km}$. In the crust of a neutron star, for instance, we have $L_{\mathrm{km}} \sim 1$. If roughly all electrons in the crust would participate in current flow, we had $N_{\mathrm{cc}} \times \sim 10^{30}$. Hence, the magnetic field strength could go up to $B \sim 10^{28}$ Gauss, a huge number compared with the maximum $B \sim 10^{15}-10^{16}$ Gauss observed in magnetars.

This crude estimate needs to be commented on in order to avoid misunderstanding. Magnetic fields are believed to be generated preferentially by dynamo actions. Such actions are presumably not at work in white dwarfs, neutron stars, magnetars or any other compact objects. The fields are produced in their differentially rotating progenitors. Take the Sun as an example with dynamo action in the convection zone of thickness $L^{\odot} \sim 2 \times 10^{5}$ $\mathrm{km}$ and average density $N_{c c}^{\odot} \sim 8 \times 10^{23}$. Using the total width of the convection zone grossly overestimates the current filament width. An absolute upper limit would be $L_{\mathrm{km}}^{\odot} \lesssim 2 \times 10^{4}$. Clearly velocities are also much less than $c$. Thus, using $c$ yields an extreme absolute upper limit on the magnetic field $B<10^{21}$ $\mathrm{T}$. The comparably strong fields in neutron stars are subsequently produced in the rapid collapse of the magnetized heavy progenitor star not having had time within the time of collapsing to dissipate the magnetic energy which becomes compressed into the tiny neutron star volume. The compression factor being of the order of $\sim 10^{12}$ yielding limit fields of $B \lesssim 10^{35}$ Gauss. 
The classical electrodynamic estimate clearly fails in providing an upper limit on magnetic field strength that would match the observational evidence.

Other no less severe discrepancies are obtained from putting the neutron star magnetic field energy equal to the total available rotational energy both in the progenitor or in the neutron star assuming equipartition of rotational and magnetic energyclearly a barely justified assumption in both cases. Magnetic energy cannot become larger than the originally available dynamical energy of its cause of which it is just a fraction. It is presumably principally questionable whether magnetic fields could ever have been produced by any classical mechanism substantially stronger than observed in neutron stars (except for a brief $\sim 10$ s long post-collapse dynamo-amplification phase at the best yielding another factor of $\sim 10-100$ [8]) and, by further concentration of magnetic energy in smaller volumes, bunching of magnetic flux tubes, as believed to occur in magnetars. If much stronger fields were generated at all, it must have happened during times and in objects where magnetic fields could have been produced by processes other than classical dynamos. One thus has to enter quantum electrodynamics respectively quantum field theory in order to infer about the principal physical limitations on the generation of any magnetic fields. The following investigation is motivated less by observations than by this fundamental theoretical question.

\section{FLUX ELEMENTS}

Quantum mechanics provides a way of obtaining a first limit on the magnetic field from solution of Schrödinger's equation, originally found by Landau [4] in 1930, of an electron orbiting in a homogeneous magnetic field. The physical interpretation of this solution was given much later in Aharonov-Bohm theory [5]. From the requirement that the magnetic flux $\Phi$ of a field $\mathbf{B}$ confined in an electron gyration orbit must be single valued, Aharonov and Bohm inferred that $\Phi=\nu \Phi_{0}$ is quantized with flux element $\Phi_{0}=2 \pi \hbar / e$, $e$ the elementary charge, and $v=1,2, \ldots$. Since $v=\Phi / \Phi_{0}$ is the number of elementary fluxes carried by the field, and $B=\Phi / \pi \ell^{2}$, putting $v=1$ defines a smallest magnetic length

$$
\ell_{B}=\left(\frac{\Phi_{0}}{\pi B}\right)^{\frac{1}{2}}=\left(\frac{2 \hbar}{e B}\right)^{\frac{1}{2}}
$$

This length, which is the gyroradius of an electron in the lowest lying Landau energy level, can be interpreted as the radius of a magnetic field line in the magnetic field $B$. Field lines become narrower the stronger the magnetic field. On the other hand, rewriting Equation (3) yields an expression for the magnetic field

$$
B_{c}=\frac{2 \hbar}{e \ell_{c}^{2}}
$$

from which, for a given shortest "critical" length $\ell_{B} \equiv \ell_{c}$ the maximum magnetic field $B_{c}$ corresponding to $\ell_{c}$ can, in principle, be estimated. Putting, for instance, $\ell_{c}=2 \pi \hbar / m c$ equal to the electron Compton length $\lambda_{0}=2 \pi \hbar / m c$, one obtains the critical pulsar (neutron star) magnetic field strength $B^{q} \equiv B_{n s} \approx$ $3 \times 10^{9} \mathrm{~T}=3 \times 10^{13}$ Gauss. It is of considerable interest that approximately this field strength was indeed inferred from observation of the fundamental $(v=1)$ electron cyclotron harmonic X-ray line detected from the HerX1 pulsar [7], roughly two decades after Aharonov and Bohm's, and half a century after Landau's theory.

\section{GENERALIZATION}

Use of the Compton wavelength relates the limiting field strength in neutron stars to quantum electrodynamics. It raises the question for a more precise theoretical determination of the quantum electrodynamic limiting field strength accounting for relativistic effects. It also raises the question whether reference to other fundamental length scales may provide other principal limits on magnetic fields if only such fields can be generated by some means, i.e., if electric currents of sufficient strengths could flow under different conditions as for instance in quantum chromodynamics.

Very formally, except as for inclusion of relativistic effects, Equation (4) provides a model equation for a limiting field in dependence on any given fundamental length scale $\ell_{c}$. Under this simplifying assumption the critical magnetic field $B_{c}$ scales simply with the inverse square of the corresponding fundamental length. Formally, this is graphically shown in Figure 1 under the assumption of validity of the Aharonov-Bohm scaling at higher energies.

The Compton limit to magnetic fields was known from straight energy considerations [cf. e.g., 8, for a review] which predict decay of the vacuum to pair formation at magnetic fields stronger than $B_{n s}$. For this reason detection of magnetic fields exceeding the quantum limit by up to three orders in magnetars was an initial surprise. However, more precise relativistic electrodynamic calculations including higher order Feynman graphs readily showed that the Compton limit can well be exceeded. To first approximation in the anomalous magnetic moment of electrons [9] the lowest Landau level shifts according to

$$
E_{L L L} \approx m c^{2}\left(1-\bar{\alpha} B / B^{q}\right)^{\frac{1}{2}}
$$

with $\bar{\alpha}=\alpha / 2 \pi$ the reduced fine structure constant. This formula is valid for $B<B^{q}$. It suggests a decrease of the lowest Landau energy level for increasing fields, obviously with violent non-physical consequences for astrophysical objects [10]. Thus, Feynman diagrams including higher-order self-attraction of electrons must be taken into account, in particular at large fields. In fields $B \gg B^{q}$ substantially exceeding $B^{q}$ the electrons become relativistically massive, and the lowest Landau level, after passing through a minimum, increases $[11,12]$ as

$$
E_{L L L} \approx m c^{2}\left\{1+\bar{\alpha}\left[\log \frac{2 B}{B^{q}}-2.077\right]^{2}+3.9 \bar{\alpha}\right\}, \quad B \gg B^{q}(6)
$$

From here it follows that the lowest Landau level energy doubles only at magnetic fields of the order of $B \sim 10^{28} \mathrm{~T}\left(\sim 10^{32}\right.$ Gauss $)$, way above any neutron star or magnetar surface magnetic fields. Relativistic self-energy corrections causing magnetic field decay will thus come into play only at these energies which may be the ultimate limit on magnetic field strengths.

It is notable that this limit approximately coincides with the [6] best recent experimental determinations of an upper limit 


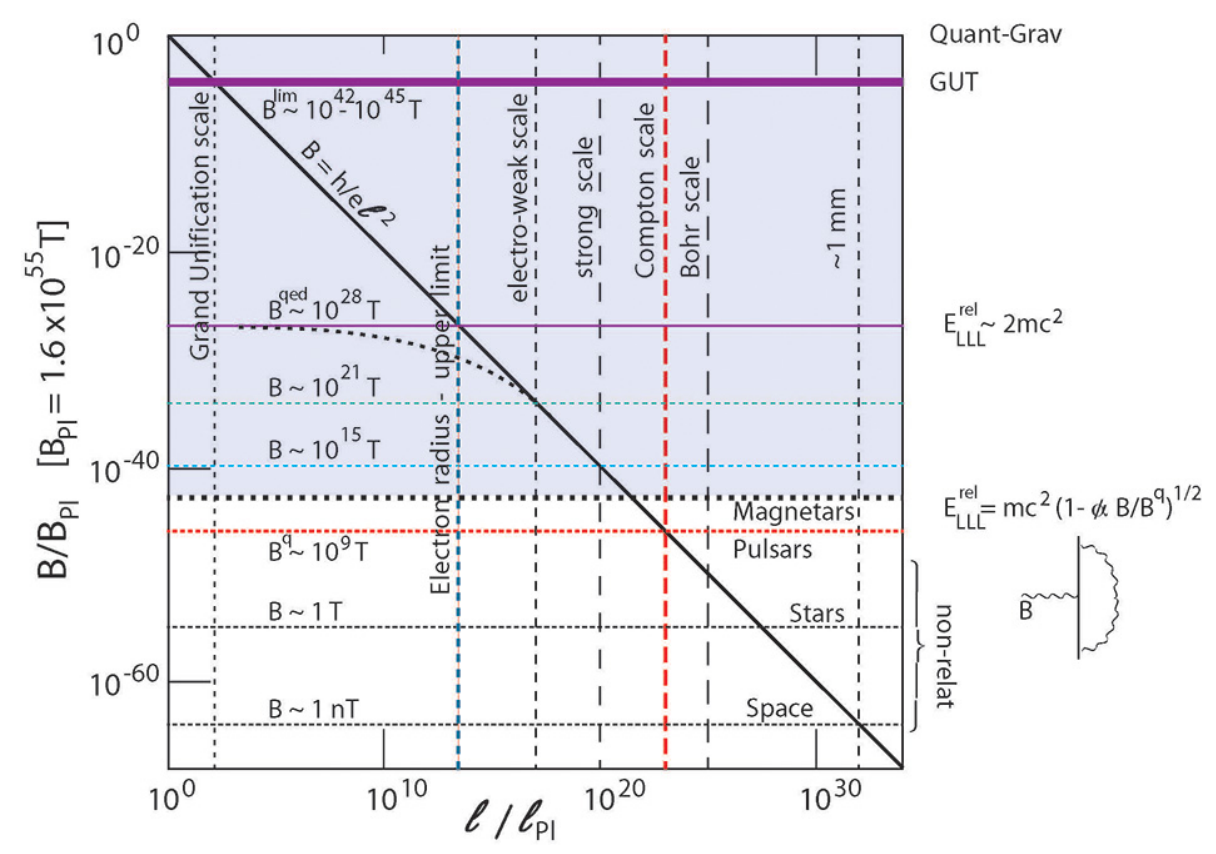

FIGURE 1 | Log-Log plot scaling of the maximum possible magnetic field strength, $B_{c}$, normalized to the (fictitious) Planck-magnetic field, $B_{P I}$, as function of fundamental length scales based on Equation (3). Length scales $\ell$ on the abscissa are normalized to the Planck length $\ell_{P 1}$. The dotted red cross indicates the crossing point of the Compton length with the Aharonov-Bohm critical magnetic field line at the so-called quantum limit field $B^{q} \approx 10^{9} \mathrm{~T}$, the critical field of magnetized neutron stars (pulsars) in agreement with observation of the strongest cyclotron lines. Horizontal lines indicate the relation between other length scales and critical magnetic fields under the assumption of validity of the Aharonov-Bohm scaling. Space magnetic fields correspond to scales of $\sim 1 \mathrm{~mm}$. Strongest detected magnetar fields correspond to the first order relativistic correction on the lowest Landau level energy $E_{L L L}$ (shown as graph on the right with $\bar{\alpha}=\alpha / 2 \pi$ the reduced fine structure constant). Inclusion of higher order corrections would allow for fields of up to $B^{\text {qed }} \sim 10^{28} \mathrm{~T}$ deep in the (shaded) relativistic domain which have not been observed. It is interesting that this limit coincides approximately with the measured [6] absolute upper limit on the electron radius (vertical blue dashed line). At GUT scales, fields could theoretically reach values up to $\sim 10^{45} \mathrm{~T}$, according to simple Aharonov-Bohm scaling. The dashed black curve indicates a possible deviation of the Aharonov-Bohm scaling near the quantum electrodynamic limit. for the electron radius. Below this scale additional effects should enter, principally inhibiting any further increasing magnetic field strengths or even existence of magnetic fields. It thus seems that up to this scale the Aharonov-Bohm scaling on which Figure 1 is based is not completely unjustified. This is most interesting also from the point of view that both the electro-weak and strong interaction scales are in the permitted domain simply since electrons maintain their nature throughout these scales. It is only the desert range of energies respectively scales which is excluded. It includes in particular the GUT range of grand unification as well as quantum gravity, domains which have played a role only in the very early universe. Any rudimentary magnetic fields from that time have been diluted by inflation and cosmological expansion to low values only $[1,2]$ located at the bottom of Figure 1.

\section{DISCUSSION AND CONCLUSIONS}

Unless magnetic monopoles ever existed and survived in the universe, magnetic fields must have been produced at any times via generation of electric currents. Fields generated in the early universe have subsequently been diluted to today's low large scale values as discussed elsewhere [1,2]. They might have been strong initially, in which case their strengths are as well subject to limitation. However, all reasonable strengths estimated from dynamo and other models in classical and chromodynamic theories [1] do most probably not reach any of the above quantum electrodynamic limits. Presumably one does not need to call for additional chromodynamic limitations. This assertion may be based on the role electrons play in current generation, which is at the base of any large scale magnetic field production. Electrons and their spins are also responsible for magnetism in solid state matter. Electrons are still believed to have no structure. In any case, at scales "inside" an electron, i.e., below the fictive electron radius $r_{e}$, currents should either loose any meaning or do not exist at all and, hence, the notion of a magnetic field will probably not make much sense anymore. One may thus believe that the upper quantum electrodynamic limit sets an absolute bound on any realistic magnetic field strengths.

The application of the Aharonov-Bohm scaling in Figure 1 to magnetic fields in the universe seems to provide a reasonable idea about the expected absolute limitations on magnetic field strengths on quantum electrodynamic scales. Clearly, the vacuum changes character at short scales and high energies, since photons become heavy switching to electroweak bosons, and quarks come into play in matter. Electrons remain the same down to at least $r_{e} \sim 10^{-22} \mathrm{~m}$, the current upper limit on the electron radius [6]. This suggests writing the critical magnetic field Equation (4) as

$$
B_{c}\left(\ell_{c}\right)=B_{\max } /\left[1+\left(\ell_{c} / \ell_{0}-1\right)^{2}\right], \quad B_{\max }=2 \hbar / e \ell_{0}^{2}
$$


where $\ell_{c} \geq \ell_{0}$, and $\ell_{0} \gtrsim r_{e}$ is the relevant minimum length above that magnetic fields make sense. In Figure 1 this behavior is indicated as the dashed black curve that deviates from the diagonal. Still, stability of the vacuum is not as clear as it is in the quantum electrodynamic range in the presence of the superstrong magnetic fields in the electro-weak and chromodynamic ranges. The problem remains that magnetic fields must have to be generated either at those small scales, or at much larger electrodynamic scales from where they collapse down to those small scales.

What concerns the generation of magnetic fields before collapse by the generally accepted dynamo or battery effects, magnetic field strengths are strictly limited by the available dynamical energies, which are far below any quantum electrodynamic limit. One may argue that, as long as the scale of the electron radius is not reached during collapse, the quantum electrodynamic scaling provides a reasonable absolute limitation on any possible magnetic field strength. Neutron stars and magnetars have scales excessively larger than the electron scale. Heavier objects by decreasing their scale could possess substantially stronger fields, but the permitted range is narrowed by the condition that such objects readily become black holes when collapsing which, by the famous no-hair theorem, do not host any magnetic fields. It is not known what would happen to the field by crossing the horizon for no information about the field would be left to the external observer. The no-hair theorem suggests that the field is simply sucked in into the hole and disappears together with the collapsing mass. Ordinary reasoning assuming maintenance of the frozen-in state then suggests that the field inside the horizon should further increase in the presumably continuing gravitational collapse.

The available strong fields which come closer to the quantum electrodynamic limits are found in neutron stars and magnetars. So far no strange star magnetic fields have been positively detected. It has even been shown [13] that such fields, possibly present in superconducting strange stars, would rotationally decay within times shorter than $\sim 20$ Myrs. In magnetars, the presence of fields stronger than $B_{n s}=B^{q}$ is now well understood [for a review of many aspects, cf., 8] as consequence of crustal effects causing local concentration of magnetic fields and extended magnetic loops bearing some similarity to the wellknown sunspots [see also the collection of articles in 3]. Effects on matter in superstrong fields were investigated first in Ruderman $[14]$ and have been reviewed in $[15,16]$ and others.

\section{REFERENCES}

1. Widrow LM, Ryu D, Schleicher DRG, Subramanian K, Tsagas CG, Treumann RA. The first magnetic fields. Space Sci Rev. (2012) 116:37-70. doi: $10.1007 / \mathrm{s} 11214-011-9833-5$
2. Ryu D, Schleicher DRG, Treumann RA, Tsagas CG, Widrow LM. Magnetic fields in the large-scale structure of the universe. Space Sci Rev. (2012) 166:135. doi: 10.1007/s11214-011-9839-z

3. Balogh A, Beskin VS, Falanga M, Lyutikov M, Mereghetti S, Piran T. The Strongest Magnetic Fields in the Universe, ISSI Space Science Series. New York, NY: Springer (in press).

4. Landau L. Diamagnetismus der Metalle. Z. Physik (1930) 64:629-37. doi: 10.1007/BF01397213

5. Aharonov Y, Bohm D. Significance of electromagnetic potentials in the quantum theory. Phys Rev. (1959) 115:485-91. doi: 10.1103/PhysRev.115.485

6. Gabrielse G, Hanneke D, Kinoshita T, Nio M, Odom B. New determination of the fine structure constant from the electron $g$ value and QED. Phys Rev Lett. (2006) 97:030802. doi: 10.1103/PhysRevLett.97.030802

7. Trümper J, Pietsch W, Reppin C, Sacco B. Evidence for strong cyclotron emission in the hard X-ray spectrum of Her X-1 (Eighth Texas Symposium on Relativistic Astrophysics). Ann NY Acad Sci. (1977) 302:538-44. doi: 10.1111/j.1749-6632.1977.tb37072.x

8. Duncan RC. Physics in ultra-strong magnetic fields. In: Gamma-ray Bursts, 5th Huntsville Symposium, Vol. 526, Huntsville, AL (2000). p. 830-41.

9. Schwinger J. On quantum-electrodynamics and the magnetic moment of the electron. Phys Rev. (1948) 73:416-7. doi: 10.1103/PhysRev.73.416

10. Chiu HL, Canuto V. Problems of intense magnetic fields in gravitational collapse. Astrophys J. (1968) 153:157-61. doi: 10.1086/180243

11. Jancovici B. Radiative correction to the ground-state energy of an electron in an intense magnetic field. Phys Rev. (1969) 187:2275-6. doi: 10.1103/PhysRev.187.2275

12. Geprägs R, Riffert H, Herold H, Ruder H, Wunner G. Electron self-energy in a homogeneous magnetic field. Phys Rev D (1994) 49:5582-9. doi: 10.1103/PhysRevD.49.5582

13. Chau HF. On the rotation and magnetic field evolution of superconducting strange stars. Astrophys J. (1997) 479:886-901. doi: 10.1086/303898

14. Ruderman M. Matter in superstrong magnetic fields. In: Hansen CJ, editor. Physics of Dense Matter, Proceedings IAU Symposium, Vol. 53. Dordrecht; Boston (1974). p. 117-31.

15. Lai D, Salpeter EE, Shapiro SL. Hydrogen molecules and chains in a superstrong magnetic field. Phys Rev A (1992) 45:4832-47. doi: 10.1103/PhysRevA.45.4832

16. Lai D. Matter in strong magnetic fields. Rev Mod Phys. (2001) 73:629-62. doi: 10.1103/RevModPhys.73.629

Conflict of Interest Statement: The authors declare that the research was conducted in the absence of any commercial or financial relationships that could be construed as a potential conflict of interest.

Received: 25 August 2014; paper pending published: 22 September 2014; accepted: 26 September 2014; published online: 16 October 2014.

Citation: Treumann RA, Baumjohann W and Balogh A (2014) The strongest magnetic fields in the universe: how strong can they become? Front. Phys. 2:59. doi: 10.3389/fphy.2014.00059

This article was submitted to Space Physics, a section of the journal Frontiers in Physics.

Copyright (C) 2014 Treumann, Baumjohann and Balogh. This is an open-access article distributed under the terms of the Creative Commons Attribution License (CC BY). The use, distribution or reproduction in other forums is permitted, provided the original author(s) or licensor are credited and that the original publication in this journal is cited, in accordance with accepted academic practice. No use, distribution or reproduction is permitted which does not comply with these terms. 